\title{
The Broadband X-Ray Spectrum of the X-Ray-obscured Type 1 AGN 2MASX J193013.80+341049.5
}

\author{
Nikita Kamraj ${ }^{1}$ (ID), Mislav Baloković, ${ }^{2,3}$, Murray Brightman ${ }^{1}$, Daniel Stern ${ }^{4}$ (ID), Fiona A. Harrison ${ }^{1}$, Roberto J. Assef ${ }^{5}$ (D), \\ Michael J. Koss ${ }^{6}$ (D), Kyuseok Oh ${ }^{7,8,10}$ (D), and Dominic J. Walton ${ }^{9}$ (D) \\ ${ }^{1}$ Cahill Center for Astronomy and Astrophysics, California Institute of Technology, Pasadena, CA 91125, USA; nkamraj@caltech.edu \\ ${ }^{2}$ Center for Astrophysics, Harvard \& Smithsonian, 60 Garden Street, Cambridge, MA 02138, USA \\ ${ }^{3}$ Black Hole Initiative at Harvard University, 20 Garden Street, Cambridge, MA 02138, USA \\ ${ }^{4}$ Jet Propulsion Laboratory, California Institute of Technology, Pasadena, CA 91109, USA \\ ${ }^{5}$ Núcleo de Astronomía de la Facultad de Ingeniería y Ciencias, Universidad Diego Portales, Av. Ejército Libertador 441, Santiago, Chile \\ ${ }^{6}$ Eureka Scientific Inc., 2452 Delmer St., Suite 100, Oakland, CA 94602, USA \\ ${ }^{7}$ Department of Astronomy, Kyoto University, Oiwake-cho, Sakyo-ku, Kyoto 606-8502, Japan \\ ${ }^{8}$ Korea Astronomy \& Space Science Institute, 776, Daedeokdae-ro, Yuseong-gu, Daejeon 34055, Republic of Korea \\ ${ }^{9}$ Institute of Astronomy, University of Cambridge, Madingley Road, Cambridge CB3 0HA, UK \\ Received 2019 September 19; revised 2019 November 4; accepted 2019 November 13; published 2019 December 26
}

\begin{abstract}
We present results from modeling the broadband X-ray spectrum of the Type 1 active galactic nucleus (AGN) 2MASX J193013.80+341049.5 using NUSTAR, Swift, and archival XMM-Newton observations. We find this source to be highly X-ray obscured, with column densities exceeding $10^{23} \mathrm{~cm}^{-2}$ across all epochs of X-ray observations, spanning an $8 \mathrm{yr}$ period. However, the source exhibits prominent broad optical emission lines, consistent with an unobscured Type 1 AGN classification. We fit the X-ray spectra with both phenomenological reflection models and physically motivated torus models to model the X-ray absorption. We examine the spectral energy distribution of this source and investigate some possible scenarios to explain the mismatch between X-ray and optical classifications. We compare the ratio of reddening to X-ray absorbing column density $\left(E_{B-V} / N_{\mathrm{H}}\right)$ and find that 2MASX J193013.80+341049.5 likely has a much lower dust-to-gas ratio relative to the Galactic interstellar medium, suggesting that the broad line region itself could provide the source of extra X-ray obscuration, being composed of low-ionization, dust-free gas.
\end{abstract}

Unified Astronomy Thesaurus concepts: Active galactic nuclei (16); Seyfert galaxies (1447); X-ray astronomy (1810)

\section{Introduction}

Under the unified model of active galactic nuclei (AGNs; Antonucci 1993; Urry \& Padovani 1995), differences between Type 1 and Type 2 AGNs arise solely from our line-of-sight viewing angle relative to a toroidal obscuring structure surrounding the central supermassive black hole (SMBH). The dusty molecular torus believed to be responsible for the obscuration seen in Type 2 AGNs likely has a clumpy distribution, as evidenced from infrared interferometric observations (Tristram et al. 2007; López-Gonzaga et al. 2016) and short-timescale variability of the line-of-sight column density in some AGNs (e.g., Marinucci et al. 2016). The unified model, while simplistic, has been successful in explaining observations of radio jets, polar ionization cones, and polarization position angle (e.g., Evans et al. 1991; Storchi-Bergmann et al. 1992).

Optical classification of AGNs is based on widths of observed emission lines. Type 1 AGNs contain both broad $\left(\right.$ FWHM $\gtrsim 1000 \mathrm{~km} \mathrm{~s}^{-1}$ ) and narrow emission lines whereas Type 2 AGNs contain only narrow emission lines. In the unified picture, this is due to obscuration of the broad line region (BLR), which is close to the central SMBH, by the molecular torus. Hence in Type 2 AGNs, only emission from the narrow line region is observed, which is extended on kiloparsec scales. In Type 1 AGNs, the torus is viewed at angles such that the line of sight to the BLR is unobscured.

X-ray classification of AGNs is typically based on measurements of the hydrogen column density $\left(N_{\mathrm{H}}\right)$. Neutral gas along

\footnotetext{
${ }^{10}$ JSPS fellow.
}

our line of sight absorbs X-ray continuum photons, with the level of absorption dependent on the column density of gas. The approximate dividing line between unobscured, Type 1 AGNs and obscured, Type 2 AGNs is at $N_{\mathrm{H}}=10^{22} \mathrm{~cm}^{-2}$ (Ricci et al. 2017). Assuming the simple picture of a static obscuring torus surrounding the AGN is correct, then there should be agreement between optical and X-ray classifications.

The Swift/BAT survey comprises all sources detected by the all-sky 14-195 keV Burst Alert Telescope (BAT) instrument onboard the Neil Gehrels Swift Observatory (Gehrels et al. 2004; Oh et al. 2018), and provides a hard-X-ray-selected sample of local AGNs that is relatively unbiased to obscuration. The Swift/BAT sample therefore offers a unique opportunity to test the unified model of AGNs. Within the Swift/BAT sample, there is excellent agreement between the optical and X-ray classifications for $\sim 95 \%$ of sources (Koss et al. 2017). However, there are a number of unusual sources in the Swift/BAT sample that show disagreement between their $\mathrm{X}$-ray and optical classifications. Some narrow-line Type 2 AGNs have been found to be X-ray unabsorbed and there is some debate about whether they represent a class of AGNs lacking a BLR (Panessa \& Bassani 2002; Tran et al. 2011; Merloni et al. 2014). In contrast, a number of Type 1 AGNs (Sy1-1.9) are highly X-ray absorbed (e.g., Shimizu et al. 2018).

In this paper, we study the spectral properties of an unusual source from the Swift/BAT survey, 2MASX J193013.80 +341049.5 , which is optically classified as a Type 1 AGN, yet is found to be highly $\mathrm{X}$-ray absorbed, with a column density $N_{\mathrm{H}}>10^{23} \mathrm{~cm}^{-2}$. The source is a nearby $(z=0.063)$ Seyfert 
Table 1

Details of the NUSTAR and XMM-Newton Observations of 2MASX J193013.80+341049.5 Considered in This Work

\begin{tabular}{|c|c|c|c|c|}
\hline Mission & Observation ID & Observation Date & $\begin{array}{l}\text { Exposure Time } \\
(\mathrm{ks})\end{array}$ & $\begin{array}{l}\text { Count Rate } \\
\left(\text { counts s }^{-1}\right)\end{array}$ \\
\hline NUSTAR & 60160713002 & 2016 Jul 19 & 23.4 & 0.142 \\
\hline NuSTAR & 60376001002 & 2017 Oct 10 & 55.4 & 0.120 \\
\hline XMM-Newton & 0602840101 & 2009 May 16 & 16.9 & 0.288 \\
\hline
\end{tabular}

Note. Observed source count rates are in the $0.5-10 \mathrm{keV}$ band for the XMM-Newton EPIC-pn detector and the 3-79 keV band for NuSTAR (FPMA).

galaxy with a bolometric luminosity $L_{\mathrm{Bol}}=1.3 \times 10^{45} \mathrm{erg} \mathrm{s}^{-1}$ (Koss et al. 2017), an $R$-band apparent magnitude of 15.8 , and a compact optical morphology. We present results from modeling the broadband X-ray spectrum of 2MASX J193013.80+341049.5 using NuSTAR, XMM-Newton, and Swift/BAT observations, and examine the multi-wavelength properties of this source. This paper is structured as follows: in Section 2 we describe the X-ray observations and data reduction procedures; in Section 3 we present results from broadband X-ray modeling; Section 4 presents analysis of multi-wavelength data on this source, including optical spectra and broadband spectral energy distributions (SEDs); Section 5 summarizes our results and presents our conclusions.

\section{X-Ray Observations and Data Reduction}

\subsection{NuSTAR}

2MASX J193013.80+341049.5 was observed with NuSTAR in 2016 July for $\sim 20 \mathrm{ks}$ as part of the Extragalactic Legacy Surveys program. ${ }^{11}$ A second, deeper NUSTAR observation was performed in 2017 October for $\sim 50 \mathrm{ks}$. Details of both these observations are presented in Table 1.

We reduced the raw event data from both NUSTAR modules, FPMA and FPMB (Harrison et al. 2013) using the NuSTAR Data Analysis Software (NuSTARDAS, version 2.14.1), distributed by the NASA High-Energy Astrophysics Archive Research Center (HEASARC) within the HEASOFT package, version 6.24. Instrumental responses were calculated based on the NuSTAR calibration database (CALDB), version 20180925. We cleaned and filtered raw event data for South Atlantic Anomaly passages using the nupipeline module. We extracted source and background spectra from the calibrated and cleaned event files using the nuproducts module. Detailed information on these data reduction procedures can be found in the NuSTAR Data Analysis Software Guide (Perri et al. 2017). A circular extraction radius of $30^{\prime \prime}$ was used for both the source and background regions. We extracted the background spectrum from source-free regions of the image on the same detector chip as the source, away from the outer edges of the field of view, which have systematically higher background. In order to maximize the available $N u S T A R$ exposure, we extracted the "spacecraft science" mode 6 data, in addition to the standard "science" mode 1 data, following the method outlined in Walton et al. (2016). We coadded the mode 1 and mode 6 spectra for each respective observation using the HEASOFT task addspec. We rebinned the spectral files using the HEASOFT task grppha to give a minimum of 20 photon counts per bin.

\footnotetext{
${ }^{11}$ https://www.nustar.caltech.edu/page/legacy_surveys
}

\subsection{Archival XMM-Newton}

In addition to the NUSTAR observations taken in the 3-79 keV band, we analyzed archival XMM-Newton observations taken in 2009 May.

We performed reduction of the XMM-Newton data using the $X M M-N e w t o n$ Science Analysis System (SAS, version 16.1.0), following the standard prescription outlined in the $X M M$ Newton ABC online guide. ${ }^{12}$ Calibrated, cleaned event files were created from the raw data files using the SAS commands epchain for the EPIC-pn detector (Strüder et al. 2001) and emchain for the two EPIC-MOS detectors (Turner et al. 2001). As recommended, we only extracted single- and doublepixel events for EPIC-pn and single- to quadruple-pixel events for EPIC-MOS. We excluded intervals of high background flux in the first $\simeq 1 / 4$ of the observation, resulting in total source exposure of 8.4, 11.0, and $12.8 \mathrm{ks}$ for the pn, MOS1 and MOS2 detectors, respectively. Source spectra were extracted from the cleaned event files using the SAS task xmmselect from a circular aperture with a radius of $40^{\prime \prime}$ centered on the source. Background spectra were extracted from a circular aperture with radius of $80^{\prime \prime}$ placed near the source on the same chip. Instrumental response files were generated for each of the detectors using the SAS tasks rmfgen and arfgen.

\subsection{Archival Swift}

In addition to the NUSTAR and XMM-Newton spectra, we also analyzed Swift/BAT spectra collected over the first 70 months of observation (Baumgartner et al. 2013), covering the $14-195 \mathrm{keV}$ band. The Swift/BAT data reduction procedure is detailed in Oh et al. (2018). We do not include Swift/XRT data in our broadband spectral modeling due to the poor signal-tonoise ratio of available Swift/XRT data, which has $<200$ total photon counts. We separately analyzed archival Swift/XRT data taken in 2005 to check for $N_{\mathrm{H}}$ variability. For this analysis, we reduced the Swift/XRT data using the XRTPIPELINE, following the standard procedures detailed in Evans et al. (2009).

\section{X-Ray Spectral Modeling}

We performed joint spectral modeling of the broadband NuSTAR, XMM-Newton, and Swift/BAT data using XSPEC v12.8.2 (Arnaud 1996). We use $\chi^{2}$ statistics for all model fitting and error estimation and we quote uncertainties at the $90 \%$ confidence level. We adopt cross-sections from Verner et al. (1996) and solar abundances from Wilms et al. (2000). We account for variability between different epochs by including cross-normalization factors between different data sets (e.g., Baloković et al. 2018). In all our modeling, we include a Galactic absorption component with a fixed column

\footnotetext{
12 https://heasarc.gsfc.nasa.gov/docs/xmm/abc/
} 

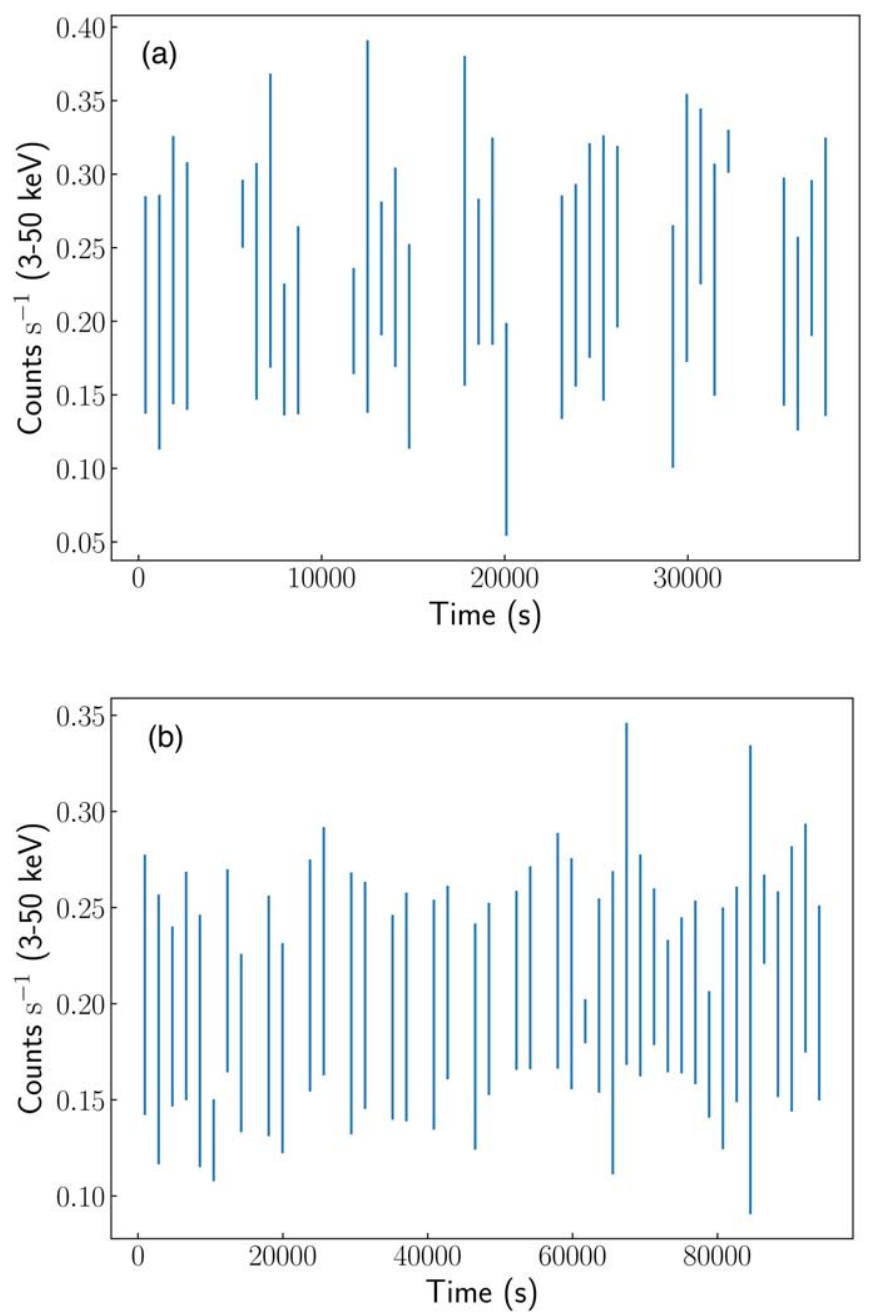

Figure 1. NUSTAR light curves of 2MASX J193013.80+341049.5 in the $3-50 \mathrm{keV}$ band, where (a) is the light curve of the $\sim 20 \mathrm{ks}$ observation taken in 2016 and (b) the deeper $\sim 50$ ks observation taken in 2017. Gaps in data are due to Earth occultation events. There is no evidence for strong X-ray variability in this source.

density of $N_{\mathrm{H}, \mathrm{Gal}}=1.62 \times 10^{21} \mathrm{~cm}^{-2}$ (Kalberla et al. 2005). We do not include NUSTAR data from the 2016 observation above $30 \mathrm{keV}$, as the source becomes background dominated above this energy, due to higher background levels relative to the 2017 observation. We model NuSTAR data from 2017 in the $3-79 \mathrm{keV}$ band, and archival XMM-Newton data in the $0.4-10 \mathrm{keV}$ band.

Analysis of the NUSTAR light curves for both observations in the $3-50 \mathrm{keV}$ band (shown in Figure 1) show little evidence for strong variability in either the 2016 or 2017 observations, justifying the use of time-averaged spectra. Flux levels in the $2-10 \mathrm{keV}$ band are also found to be similar between the two observations. Hogg et al. (2012) performed a detailed light curve analysis of the archival XMM-Newton observation and found no significant variability in the XMM-Newton EPIC-pn and MOS light curves.

In our spectral modeling, we simultaneously fit the $X M M$ Newton (EPIC-pn and EPIC-MOS), NuSTAR (FPMA and FPMB), and $S$ wift/BAT spectra, covering a total energy range from 0.4 to $150 \mathrm{keV}$. We begin our analysis by fitting a simple absorbed cutoff power-law model to the broadband data. As shown in Figure 2, an $\mathrm{Fe} \mathrm{K} \alpha$ emission line near $6.4 \mathrm{keV}$ is evident in addition to excess soft emission below $3 \mathrm{keV}$.

We construct models consisting of both phenomenological reflection models and physically motivated torus models. All models include a photoelectric neutral absorber, power-law continuum, and a scattered power-law continuum. We leave the line-of-sight column density $N_{\mathrm{H}}$ free between different epochs. We list the applied models in XSPEC notation as follows.

1. phabs $\times$ (zphabs $\times$ cabs $\times$ cutoffpl + zgauss + pexrav + constant $\times$ cutoffpl): Models an absorbed cutoff power law with a Gaussian $\mathrm{Fe} \mathrm{K} \alpha$ line, a cold Compton reflection component and a scattered power-law component. phabs models Galactic absorption while zphabs models host galaxy absorption. pexrav (Magdziarz \& Zdziarski 1995) models reflection off a slab of infinite extent and optical depth covering between 0 and $2 \pi$ sr of the sky relative to the illuminating source, corresponding to $R$ between 0 and 1 .

2. phabs $\times$ (cabs $\times$ TBFeo $\times$ cutoffpl + pexmon + constant $\times$ cutoffpl): Similar to Model 1, with the exception that zphabs is replaced with TBFeo, which models absorption with a variable iron abundance; the zgauss and pexrav components are replaced with pexmon, which is a slab reflection model that self consistently models the $\mathrm{Fe}$ and $\mathrm{Ni} \mathrm{K}$ complexes and Compton reflection hump assuming a semi-infinite plane geometry.

3. phabs $\times($ cabs $\times$ TBFeo $\times$ cutoffpl + borus $02+$ constant $\times$ cutoffpl): In the physically motivated borus 02 model (Baloković et al. 2018), obscuring material is arranged in a toroidal structure around the central AGN, with a variable opening angle and torus column density that can be decoupled from the line-of-sight column density. This model provides self-consistent modeling of the fluorescent line emission and Compton reflection features.

For Model 1, we set iron and light element abundances to solar values and fix the inclination angle of the plane of reflecting material at the default value of $\cos \theta=0.45$. We tie the photon index and normalization of the reflected power law to that of the incident power law. We fix the energy of the $\mathrm{Fe}$ $\mathrm{K} \alpha$ line at $6.4 \mathrm{keV}$. As shown in Figure 2(c), this model provides a considerable improvement in the fit compared to an absorbed cutoff power-law model, with $\chi^{2} /$ dof $=826 / 798$. We find evidence of moderate reflection, with the reflection parameter $R$ constrained to $0.26_{-0.23}^{+0.32}$. The cutoff energy is found to be strikingly low; $E_{\text {cut }}=50_{-11}^{+19} \mathrm{keV}$. We find a fairly hard photon index $\left(\Gamma=1.35_{-0.15}^{+0.18}\right)$, which may partly account for the low cutoff energy obtained, due to degeneracy between $E_{\text {cut }}$ and $\Gamma$, as evident in Figure 4 . The line-of-sight column density exceeds $10^{23} \mathrm{~cm}^{-2}$ in all epochs of observation, confirming this source to be highly X-ray absorbed. The value of $N_{\mathrm{H}}$ was similar between the NUSTAR observations, at $\sim(3-4) \times 10^{23} \mathrm{~cm}^{-2}$. For the archival XMM-Newton observation, $N_{\mathrm{H}}$ was slightly lower at $\sim 2.6 \times 10^{23} \mathrm{~cm}^{-2}$, but still consistent with high $\mathrm{X}$-ray obscuration, and with the values reported by Hogg et al. (2012). We also examined archival Swift/XRT spectra taken in 2005 December and found high levels of absorption, with $N_{\mathrm{H}}$ found to be $5.3_{-2.3}^{+5.5} \times 10^{23} \mathrm{~cm}^{-2}$ from fitting Model 1 to the data. 


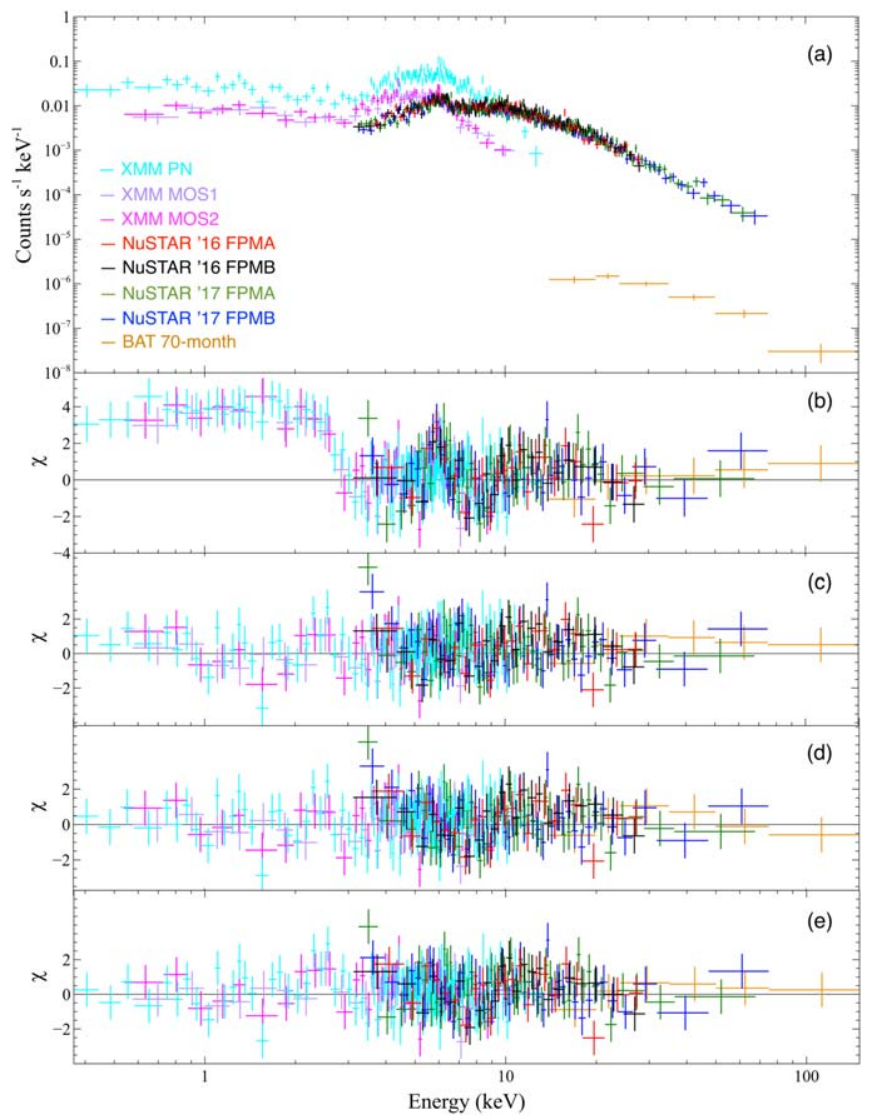

Figure 2. Broadband X-ray spectrum of 2MASX J19301380+3410495 (a), alongside fit residuals for (b) an absorbed cutoff power-law model $\left(\chi^{2}\right)$ dof $=1409 / 811$ ), (c) absorbed cutoff power law with an Fe K $\alpha$ line, scattered power law, and a reflection component incorporated using the pexrav model $\left(\chi^{2} /\right.$ dof $\left.=826 / 798\right)$, (d) absorbed cutoff power law with a variable iron abundance absorber and reflection modeled with pexmon $\left(\chi^{2} /\right.$ dof $=816 /$ $798)$, and (e) borus model $\left(\chi^{2} /\right.$ dof $\left.=808 / 795\right)$.

We find a fairly low equivalent width of the $\mathrm{Fe} \mathrm{K} \alpha$ line, at $\approx 80 \mathrm{eV}$ for the XMM-Newton observation and $\approx 90 \mathrm{eV}$ for the NUSTAR observation. This motivates the exploration of alternative models with a variable iron abundance. In Model 2, we allow the iron abundance to vary by replacing the photoelectric absorption component zphabs with an absorber with variable iron abundance (TBFeo). We also replace the pexrav reflection model and zgauss line component with the phenomenological pexmon model, which self-consistently models both the reflection continuum and the $\mathrm{Fe} \mathrm{K} \alpha$ line. We tie the iron abundance parameter of the pexmon model to that of TBFeo. Model 2 yields an improvement to the fit compared to Model 1 , with $\chi^{2} /$ dof $=816 / 798$. However, we find that the iron abundance is consistent with the solar value when left as a free parameter. The reduction in $\chi^{2} /$ dof compared to Model 1 is likely attributed to differences between the two models, such as the inclusion of additional emission lines in Model 2. Furthermore, Model 2 produces slightly higher $N_{\mathrm{H}}$ values for a given epoch (see Table 2).

The phenomenological models used to account for reflection assume a simplistic slab geometry of the reflector with infinite extent and optical depth. While such models provide a convenient, simplified picture of the AGN reprocessor, they are not a physically realistic description of the geometry of the circumnuclear material, which is thought to have a roughly toroidal shape. We thus construct a third, physically motivated model in which the pexmon component is replaced by borus02 (Baloković et al. 2018). The borus02 model self-consistently computes the absorbed and reprocessed emission for a torus geometry with a central illuminating $\mathrm{X}$-ray source. It provides more flexibility in modeling the torus geometry compared to previously developed torus models such as MYTorus (Murphy \& Yaqoob 2009), as it includes the opening angle, the high-energy cutoff, and the relative iron abundance as free parameters. In contrast, MYTorus assumes a uniform density torus with a fixed opening angle of $60^{\circ}$, solar abundance of iron and a termination energy at $500 \mathrm{keV}$. Both models can emulate torus clumpiness by allowing the average column density through the torus to be independent of the lineof-sight column density.

With Model 3, we set the covering factor of the torus, $C_{f}$, equal to $\cos i$, where $i$ is the viewing angle of the torus. We find the fit naturally converges close to $C_{f}=\cos i$ when both parameters are left free to vary. We tie the iron abundance parameter of borus02 to that of TBFeo. We link the photon index and normalization of the borus 02 component to that of the incident power law. Model 3 provides the best fit to the data compared to Models 1 and 2, with $\chi^{2} / \operatorname{dof}=808 / 795$. Leaving the iron abundance free results in a sub-solar value $\left(A_{\mathrm{Fe}}=0.45_{-0.05}^{+0.06}\right)$, consistent with the weak $\mathrm{Fe} \mathrm{K} \alpha$ line observed in this source.

Table 2 summarizes our modeling results for some of the key best-fit parameters for each of the three spectral models applied in this work. We conclude from our broadband spectral fitting that our physically motivated borus02 model (Model 3) provides the best fit to the data and also gives a photon index that lies within the typical range observed for Seyferts. We show the full broadband unfolded X-ray spectrum for the borus 02 model fit in Figure 3.

With the additional, deeper $50 \mathrm{ks}$ NuSTAR observations taken in 2017, the high-energy cutoff of the X-ray continuum is confirmed to be constrained to values that are atypically low compared to the Seyfert population. Ricci et al. (2017) found that the median cutoff energy for the Swift/BAT sample of unobscured AGNs is $E_{\text {cut }}=210 \pm 36 \mathrm{keV}$. Figure 4 shows the contour plot of the photon index against the high-energy cutoff from the borus 02 model fit. While there is some degree of degeneracy between these two parameters, the value of $E_{\text {cut }}$ is constrained to low values that are below the median cutoff energy found by Ricci et al. (2017). Such a low coronal cutoff is unusual but not unprecedented, with recent detections reported of AGNs with high-energy cutoffs within the NuSTAR band (e.g., Kara et al. 2017; Tortosa et al. 2017; Kamraj et al. 2018).

Various explanations have been proposed for the origin of low-temperature coronae. For sources accreting at high Eddington rates, Compton cooling may be enhanced due to the stronger radiation field present in comparison to lower Eddington ratio Seyferts (Kara et al. 2017). For AGNs accreting well below the Eddington limit, low coronal temperatures may be attributed to a high optical depth within the corona, which results in more effective cooling due to multiple inverse Compton scatterings of seed photons from the accretion disk (Tortosa et al. 2017). Low temperatures can also be achieved if the corona consists of a hybridized plasma, containing both thermal and non-thermal particles (e.g., Ghisellini et al. 1993; Zdziarski et al. 1993; Fabian et al. 2017). In such a hybridized system, only a small fraction of non-thermal electrons with energies above $1 \mathrm{MeV}$ are needed 


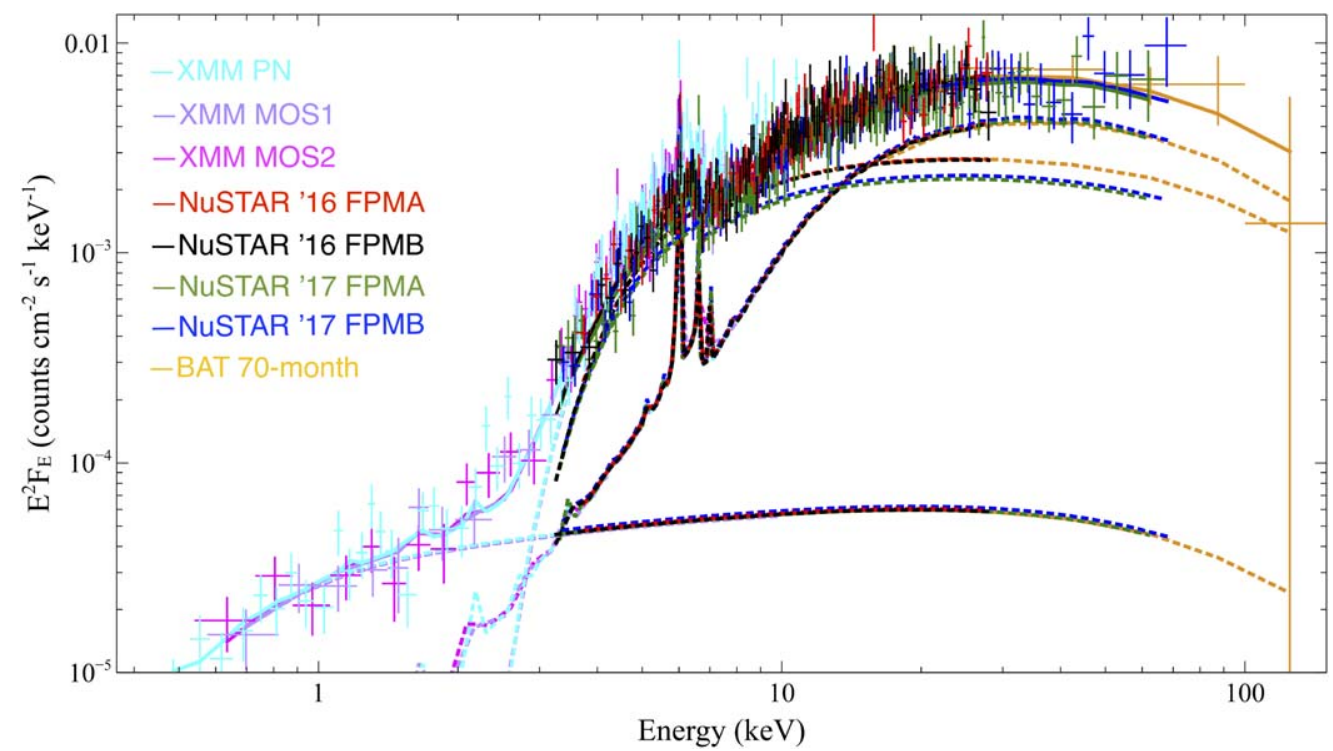

Figure 3. Broadband X-ray spectrum of 2MASX J193013.80+341049.5 unfolded through the borus02 model (Model 3). Solid lines represent total model while dashed lines depict individual model components.

Table 2

Best-fit Parameter Values from Modeling the Broadband X-Ray Spectrum of 2MASX J193013.80+341049.5

\begin{tabular}{|c|c|c|c|c|c|}
\hline Model Component & Parameter & & Model 1 & Model 2 & Model 3 \\
\hline \multirow[t]{3}{*}{ zphabs } & $N_{\mathrm{H}}(N u S T A R 2016)$ & {$\left[10^{23} \mathrm{~cm}^{-2}\right]$} & $3.3 \pm 0.3$ & $\ldots$ & $\ldots$ \\
\hline & $N_{\mathrm{H}}(N u S T A R 2017)$ & {$\left[10^{23} \mathrm{~cm}^{-2}\right]$} & $3.8 \pm 0.3$ & $\ldots$ & $\cdots$ \\
\hline & $N_{\mathrm{H}}(X M M-N e w t o n)$ & {$\left[10^{23} \mathrm{~cm}^{-2}\right]$} & $2.6_{-0.2}^{+0.3}$ & $\ldots$ & $\ldots$ \\
\hline \multirow[t]{4}{*}{ TBFeo } & $A_{\mathrm{Fe}}$ & [solar] & $\ldots$ & $1.07_{-0.30}^{+0.25}$ & $0.45_{-0.05}^{+0.06}$ \\
\hline & $N_{\mathrm{H}}(N u S T A R 2016)$ & {$\left[10^{23} \mathrm{~cm}^{-2}\right]$} & $\ldots$ & $4.8_{-0.5}^{+0.6}$ & $5.2_{-0.1}^{+0.5}$ \\
\hline & $N_{\mathrm{H}}(N u S T A R 2017)$ & {$\left[10^{23} \mathrm{~cm}^{-2}\right]$} & $\ldots$ & $5.5_{-0.5}^{+0.7}$ & $4.9_{-0.7}^{+0.5}$ \\
\hline & $N_{\mathrm{H}}(X M M-N e w t o n)$ & {$\left[10^{23} \mathrm{~cm}^{-2}\right]$} & $\ldots$ & $3.9_{-0.3}^{+0.4}$ & $4.7_{-0.4}^{+0.3}$ \\
\hline \multirow[t]{5}{*}{ pexrav } & $\Gamma^{\mathrm{a}}$ & & $1.35_{-0.15}^{+0.18}$ & $\ldots$ & $\ldots$ \\
\hline & $E_{\text {cut }}$ & {$[\mathrm{keV}]$} & $49.9_{-11.2}^{+19.0}$ & $\ldots$ & $\ldots$ \\
\hline & $R$ & & $0.26_{-0.23}^{+0.32}$ & $\ldots$ & $\ldots$ \\
\hline & $A_{\mathrm{Fe}}$ & [solar] & 1 (fixed) & $\ldots$ & $\ldots$ \\
\hline & Norm $^{b}$ & {$\left[10^{-3}\right]$} & $1.41_{-0.37}^{+0.59}$ & $\ldots$ & $\ldots$ \\
\hline \multirow[t]{4}{*}{ pexmon } & $\Gamma^{\mathrm{a}}$ & & $\ldots$ & $1.33_{-0.14}^{+0.21}$ & $\ldots$ \\
\hline & $E_{\text {cut }}$ & {$[\mathrm{keV}]$} & $\ldots$ & $49.9_{-13.2}^{+38.3}$ & $\ldots$ \\
\hline & $R$ & & $\ldots$ & $0.25_{-0.10}^{+0.23}$ & $\ldots$ \\
\hline & Norm $^{b}$ & {$\left[10^{-3}\right]$} & $\ldots$ & $1.47_{-0.34}^{+0.54}$ & $\ldots$ \\
\hline \multirow[t]{5}{*}{ borus 02} & $\Gamma^{\mathrm{a}}$ & & $\ldots$ & $\ldots$ & $1.73_{-0.27}^{+0.05}$ \\
\hline & $E_{\text {cut }}$ & {$[\mathrm{keV}]$} & $\ldots$ & $\ldots$ & $71.9_{-41.0}^{+21.2}$ \\
\hline & $\log N_{\mathrm{H}, \text { Tor }}$ & & $\ldots$ & $\ldots$ & $24.25_{-0.12}^{+0.02}$ \\
\hline & $C_{f}^{\mathrm{c}}$ & {$[\%]$} & $\ldots$ & $\ldots$ & $>83.9$ \\
\hline & Norm $^{\text {b }}$ & {$\left[10^{-3}\right]$} & $\ldots$ & $\ldots$ & $2.44_{-0.81}^{+0.63}$ \\
\hline$\chi^{2} /$ dof & & & $826 / 798$ & $816 / 798$ & $808 / 795$ \\
\hline
\end{tabular}

Notes.

${ }^{\text {a }}$ Continuum photon index.

b Power-law normalization in units of counts $\mathrm{s}^{-1} \mathrm{keV}^{-1}$ at $1 \mathrm{keV}$.

$\mathrm{c}$ Torus covering factor.

to result in runaway electron-positron pair production. The cooled pairs redistribute their available energy, thereby reducing the mean energy per particle and decreasing the coronal temperature. Such cooling would produce a hard nonthermal tail in the X-ray spectrum and an annihilation feature at $511 \mathrm{keV}$, both of which are currently undetectable with current $\mathrm{X}$-ray instrumentation. In order to robustly test hybrid plasma models, next-generation hard X-ray observatories with high sensitivity at energies beyond $100 \mathrm{keV}$, such as the HighEnergy X-ray Probe (Madsen et al. 2018), will be essential.

\section{Multi-wavelength Analysis}

From broadband X-ray spectral modeling, we confirm that 2MASX J193013.80+341049.5 exhibits high levels of X-ray 
Table 3

Observation Details of the Optical Spectra of 2MASX J193013.80+341049.5 Considered in This Work

\begin{tabular}{|c|c|c|c|c|c|c|}
\hline Observation Date & Telescope & Instrument & $\begin{array}{c}\text { Exposure Time } \\
\text { (s) }\end{array}$ & $\begin{array}{l}\text { Slit Size } \\
\qquad\left({ }^{\prime \prime}\right)\end{array}$ & $\begin{array}{l}\text { Slit Size } \\
\quad(\mathrm{kpc})\end{array}$ & $\begin{array}{c}\text { Spectral Resolution (FWHM) } \\
(\AA)\end{array}$ \\
\hline 2006 May 8 & Orzale & BFOSC & 3600 & 2 & 2.4 & 13 \\
\hline 2007 Jun 16 & KPNO & Goldcam & 3600 & 2 & 2.4 & 3.3 \\
\hline 2016 Oct 2 & Palomar & DBSP & 300 & 1.5 & 1.8 & $4.2,5.9$ \\
\hline 2018 Aug 11 & Palomar & DBSP & 300 & 2 & 2.4 & $4.8,7.4$ \\
\hline 2019 Aug 28 & Palomar & DBSP & 900 & 1.5 & 1.8 & $4.4,5.8$ \\
\hline
\end{tabular}

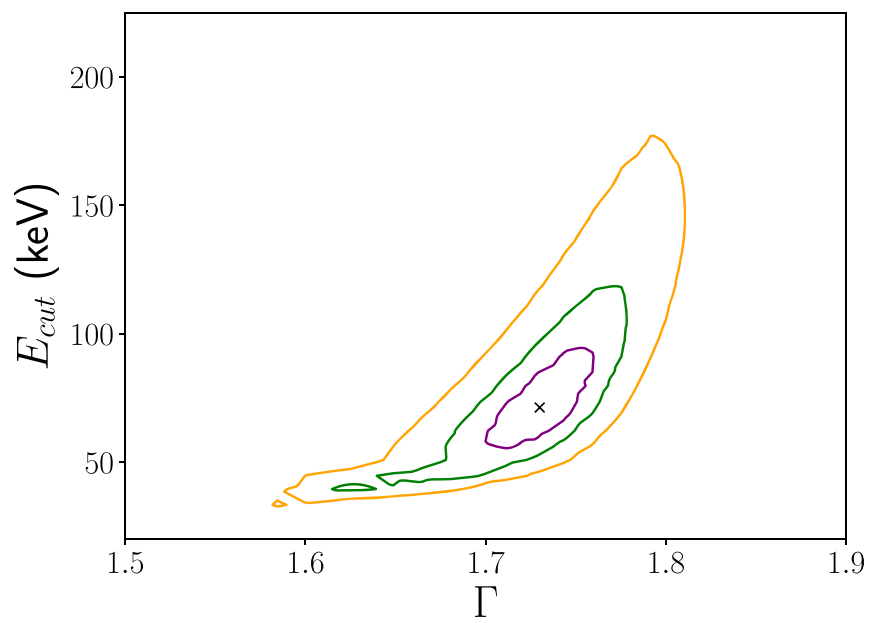

Figure 4. $E_{\text {cut }}-\Gamma$ contour plot from the borus 02 model fit (Model 3) to broadband X-ray data of 2MASX J193013.80+341049.5. The solid purple, green, and yellow contours correspond to the $68 \%, 90 \%$, and $99 \%$ confidence levels, respectively. The black cross represents the best-fit values of the parameters from applying the borus 02 model.

obscuration, with $N_{\mathrm{H}}$ exceeding $10^{23} \mathrm{~cm}^{-2}$ in both the NuSTAR and archival XMM-Newton observations. The X-ray spectral features of this source are characteristic of a classic obscured, Type 2 AGN. The high X-ray absorption present is thus in clear conflict with the optical classification of this source as a Type 1 AGN. We explore properties of this enigmatic source at other wavelengths to investigate whether there are other unusual features possibly linked to the high X-ray obscuration but comparatively lower optical obscuration present in this source. We also discuss possible mechanisms for producing such mismatches between X-ray and optical classifications.

\subsection{Optical Spectra}

2MASX J193013.80+341049.5 has consistently been classified as a Type 1 AGN from optical spectra taken over several epochs (e.g., Landi et al. 2007; Trippe et al. 2011), which show clear broad components to the $\mathrm{H} \alpha$ and $\mathrm{H} \beta$ lines. We also obtained recent optical spectra using the Double Spectrograph (DBSP) instrument on the 200 inch Hale telescope at Palomar Observatory. Observations were performed in 2016 October, 2018 August, and 2019 August. We compare the Palomar spectra with those taken at Orzale in 2006 and KPNO in 2007 (Koss et al. 2017). Full observation details of the optical spectra examined in this work are presented in Table 3. Broad $\mathrm{H} \alpha$ and $\mathrm{H} \beta$ lines are present in all epochs, consistent with a Type 1 optical classification (Figure 5). We also observed a nearby galaxy companion during the UT 2019 August 28 observation, 2MASS J193015.12+34111.18, which is $27.3^{\prime \prime}$ to the northeast of the AGN (33.4 kpc). The galaxy is an absorption line system at a close redshift $(z=0.063$ based on the $\mathrm{Ca} \mathrm{H}+\mathrm{K}$ absorption lines) to the primary AGN galaxy.

For emission line measurements, we follow the procedure used in the OSSY database (Oh et al. 2011) and its broad-line prescription (Oh et al. 2015). We apply stellar templates (Bruzual \& Charlot 2003; Sánchez et al. 2006) and emissionline fitting in a rest-frame ranging from $3780 \AA$ to $7580 \AA$. Using the broad $\mathrm{H} \alpha$ flux and line widths from the 2016 observation, we derive a black hole mass of $\log \left(M_{\mathrm{BH}} / M_{\odot}\right)=8.3$ using the virial approximation of Greene \& Ho (2007). The flux of the broad $\mathrm{H} \beta$ line, broad $\mathrm{H} \alpha$ line, and continuum (4760-4780 $\AA$ ) are observed to decrease over the period 2006-2019 (Table 4) by more than a factor of 2. Errors are dominated by flux calibration of the order $10 \%-20 \%$. The $\mathrm{H} \beta$ line FWHM remains roughly constant across observations. It is unlikely that the mismatch in X-ray and optical classifications is due to short timescale variability of the line-of-sight absorption, as $N_{\mathrm{H}}$ remains consistently high in all epochs of X-ray observations. Furthermore, Hogg et al. (2012) note little variability in optical spectra taken three months apart (Landi et al. 2007; Winter et al. 2010).

\subsection{X-Ray to Mid-IR Relation}

It is well-established that the X-ray and mid-IR emission from AGNs are correlated (e.g., Lutz et al. 2004; Gandhi et al. 2009; Stern 2015). We investigate whether 2MASX $\mathrm{J} 193013.80+341049.5$ has mid-IR properties that are consistent with the observed correlation between mid-IR and X-ray luminosity. We estimate the mid-IR luminosity at $6 \mu \mathrm{m}$ by constructing the broadband SED for 2MASX J193013.80 +341049.5 using publicly available data from the Vizier catalog. The data obtained from Vizier includes measurements from catalogs such as 2MASS, Gaia, PanSTARRS, AKARI, Wide-field Infrared Survey Explorer and XMM-UVOT, covering a total wavelength range from 0.3 to $100 \mu \mathrm{m}$. We linearly interpolate between 3.75 and $15 \mu \mathrm{m}$ to determine the rest-frame $6 \mu \mathrm{m}$ flux density. We convert from specific flux to luminosity using a luminosity distance of $268 \mathrm{Mpc}$ obtained from the NASA/IPAC Extragalactic Database. We calculate the X-ray luminosity from the unabsorbed flux in the $2-10 \mathrm{keV}$ band, using the $2017 \mathrm{NUSTAR}$ data. We also estimate the predicted $2-10 \mathrm{keV}$ luminosity based on the $\mathrm{H} \alpha$ luminosity measured from the the 2016 optical spectra, using the observed relation between intrinsic $2-10 \mathrm{keV}$ luminosity and $\mathrm{H} \alpha$ luminosity (Panessa et al. 2006). We find the predicted X-ray luminosity to be consistent with the intrinsic luminosity observed from the NuSTAR data.

Figure 6 shows the rest-frame $2-10 \mathrm{keV}$ luminosity against the rest-frame $6 \mu \mathrm{m}$ luminosity for 2MASX J193013.80 +341049.5 , along with correlations reported in the literature. Fiore et al. (2009) report a mid-IR to X-ray correlation based 

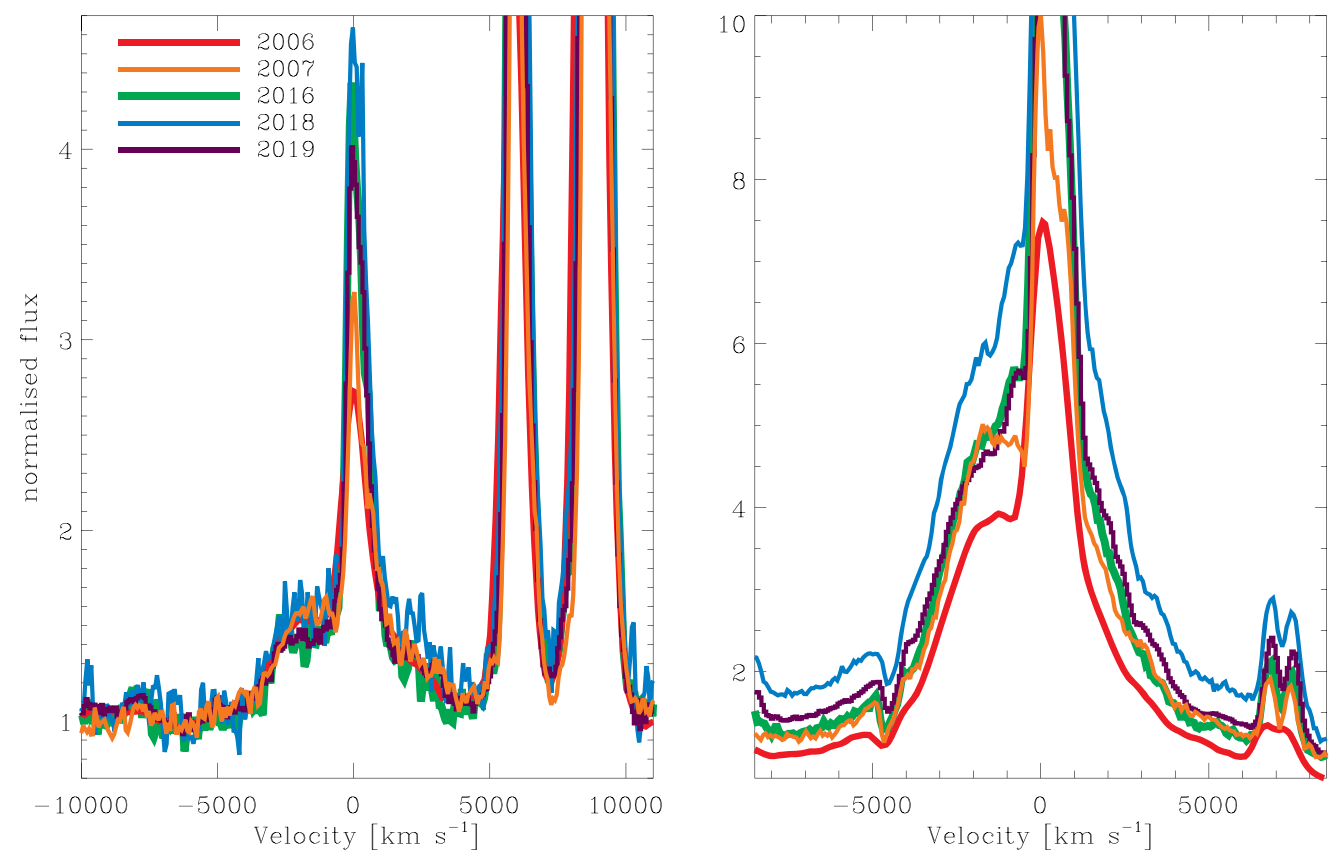

Figure 5. Optical spectra of 2MASX J193013.80+341049.5 taken at Orzale (2006), KPNO (2007), and Palomar Observatory (2016, 2018, 2019). The fluxes have been normalized by the emission in a line-free region (4760-4780 $\mathrm{A})$. Left and right panels show the velocity centered on the $\mathrm{H} \beta$ and $\mathrm{H} \alpha$ line profiles respectively.

Table 4

Flux Details and Selected Line-width Properties of the Optical Spectra of 2MASX J193013.80+341049.5 Considered in This Work

\begin{tabular}{lcccr}
\hline \hline Observation Date & $\begin{array}{c}\text { Continuum Flux } \\
\left(\mathrm{erg} \mathrm{s}^{-1} \mathrm{~cm}^{-2} \AA^{-1}\right)\end{array}$ & $\begin{array}{c}\mathrm{H} \alpha \text { Flux } \\
\left(10^{-17} \mathrm{erg} \mathrm{s}^{-1} \mathrm{~cm}^{-2}\right)\end{array}$ & $\begin{array}{c}\mathrm{H} \alpha \text { FWHM } \\
\left(\mathrm{km} \mathrm{s}^{-1}\right)\end{array}$ & $\begin{array}{c}\mathrm{H} \beta \text { Flux } \\
\left(10^{-17} \mathrm{erg} \mathrm{s}^{-1} \mathrm{~cm}^{-2}\right)\end{array}$ \\
\hline 2006 May 8 & 160 & 60501 & 6948 & 8580 \\
2007 Jun 16 & 77 & 37097 & 7053 & 4363 \\
2016 Oct 2 & 78 & 41239 & 6720 & 3189 \\
2018 Aug 11 & 48 & 33022 & 6697 & 3577 \\
2019 Aug 28 & 58 & 30915 & 7227 & 3373 \\
\end{tabular}

Note.

${ }^{\text {a }}$ Continuum flux density estimated in the $4760-4780 \AA$ A band.

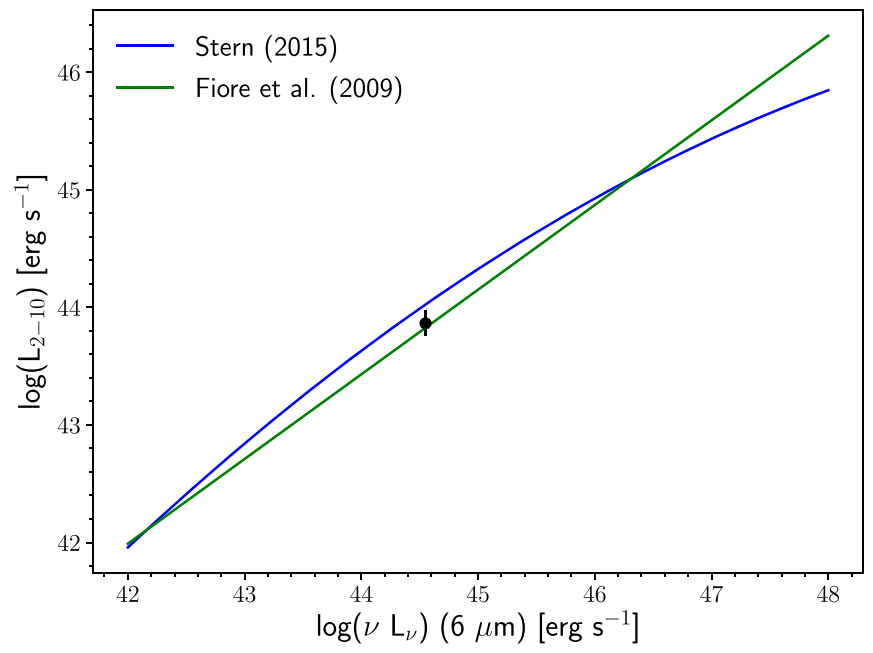

Figure 6. Rest-frame $2-10 \mathrm{keV}$ X-ray luminosity against rest-frame $6 \mu \mathrm{m}$ midinfrared luminosity for 2MASX J193013.80+341049.5 (black point) along with published relations from Fiore et al. (2009) and Stern (2015).

on samples of X-ray-selected Type 1 AGNs in the COSMOS and CDFS fields. The relation presented in Stern (2015) was obtained by including a sample of the most luminous quasars from the Sloan Digital Sky Survey, and is appropriate for AGNs across a large luminosity range, spanning from the Seyfert through to the quasar regime. The location of our source is consistent with both these published relations, indicating that 2MASX J193013.80+341049.5 is not atypical in terms of its mid-IR/X-ray luminosity ratio.

\subsection{Broadband SED and $\alpha_{\mathrm{ox}}$}

We construct the broadband SED for 2MASX J193013.80 +341049.5 using publicly available flux densities from the Vizier catalog and PanSTARRS DR2. We perform SED fitting in order to investigate the relationship between UV and X-ray luminosity for this source, parameterized by the $\alpha_{\mathrm{ox}}$ spectral slope, defined as:

$$
\alpha_{\mathrm{ox}}=-0.384 \times \log \left[L_{2 \mathrm{keV}} / L_{2500}\right]
$$

where $L_{2 \mathrm{keV}}$ and $L_{2500}$ are the monochromatic luminosities at $2 \mathrm{keV}$ and $2500 \AA$, respectively. We determine the monochromatic luminosity at $2500 \AA$, corrected for dust-reddening, via SED template fitting. We model the SED of 2MASX $\mathrm{J} 193013.80+341049.5$ in the $0.03-30 \mu \mathrm{m}$ range using the algorithm and empirical templates of Assef et al. (2010). The models consist of a linear combination of a dust-reddened 


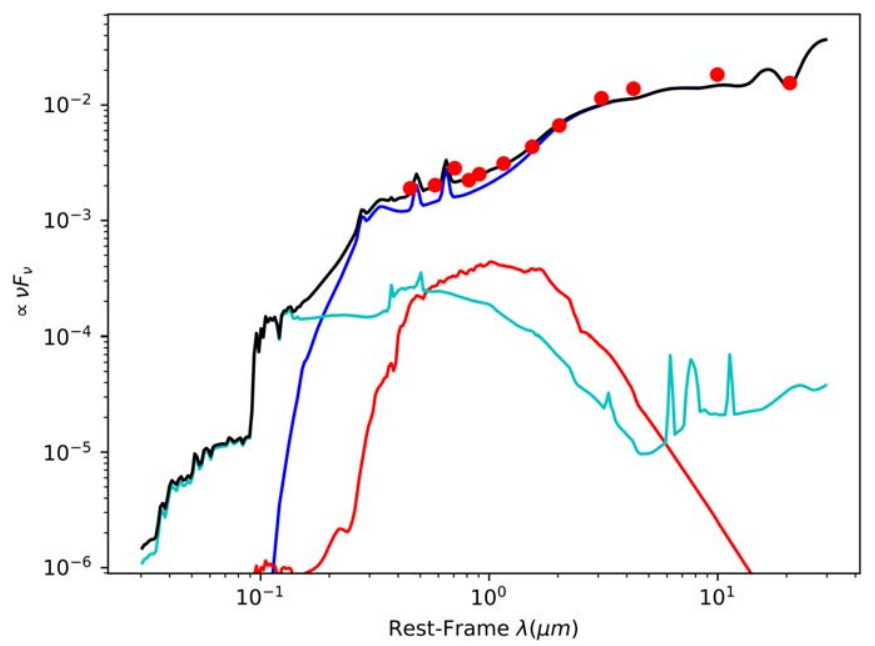

Figure 7. Spectral energy distribution (SED) of 2MASX J193013.80 +341049.5 . The best-fit SED model (black line) consists of an AGN component (blue line), a young stellar population (cyan line), and an old stellar population (red line). Solid red points are observed flux densities used for the SED fit, obtained from publicly available data from the Vizier catalog and PanSTARRS DR2.

AGN template and three empirical galaxy templates, corresponding to E-, Sbc-, and Im-type galaxies. We do not include UV data in our SED fitting due to the uncertainty in the UV extinction correction. Figure 7 presents the best-fit SED model of 2MASX J193013.80+341049.5 along with individual model components. We linearly interpolate the best-fit, unreddened AGN component to determine the intrinsic specific flux at $2500 \AA$. We compute the monochromatic X-ray luminosity at $2 \mathrm{keV}$ from modeling the broadband X-ray spectra, correcting for absorption.

In Figure 8 we present the monochromatic X-ray luminosity as a function of the monochromatic UV luminosity, along with fitted relations reported in the literature. Lusso et al. (2010) present a significant correlation between $L_{2 \mathrm{keV}}$ and $L_{2500}$ based on a sample of $545 \mathrm{X}$-ray-selected Type 1 AGNs from the XMM-COSMOS survey, spanning a wide range of redshifts and X-ray luminosities. In Lusso \& Risaliti (2016), a tighter correlation is reported using a sample of 2685 quasars that have been optically selected with homogeneous SED and X-ray detections, with dust-reddened and gas-obscured sources excluded from the sample. 2MASX J193013.80+341049.5 is consistent with observed relations between $L_{2 \mathrm{keV}}$ and $L_{2500}$ (Figure 8), indicating that this source is also not atypical in terms of its $\mathrm{UV} / \mathrm{X}$-ray luminosity ratio. We find $\alpha_{\mathrm{ox}} \sim 1.4$, which is within the typical range of $\alpha_{\mathrm{ox}}$ distributions, which covers 1.2-1.8 (Lusso et al. 2010).

The normal X-ray-to-optical ratio (parameterized by $\alpha_{\mathrm{ox}}$ ) of 2MASX J193013.80+341049.5 indicates that it is unlikely that the Type 1 optical classification is due to scattering of BLR photons into our line of sight through a region of lower column density. If there were strong scattering present, a stronger X-ray-to-optical ratio would be expected due to suppression of optical emission. We further rule out a scattering scenario through our broadband SED fitting, where we allow for a second unobscured AGN component to account for the scattered light leaked from a primary obscured AGN component. The best fit for such a model produces zero flux for the

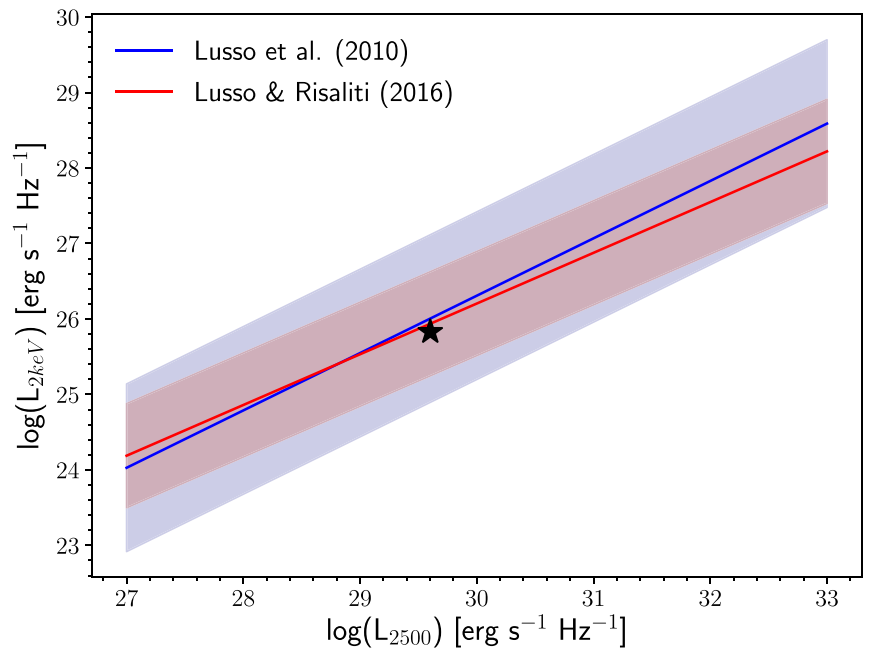

Figure 8. Rest-frame monochromatic $2 \mathrm{keV}$ luminosity $L_{2 \mathrm{keV}}$, against restframe $2500 \AA$ luminosity $L_{2500}$ for 2MASX J193013.80+341049.5 (black star). Also plotted are literature relations from Lusso et al. (2010) and Lusso \& Risaliti (2016). The shaded regions represent the $3 \sigma$ dispersion in the fitted relations.

second AGN component, indicating no evidence for scattered/ reflected light leaking into our line of sight.

\subsection{Gas-to-dust Ratio}

Some studies have reported Type 1 AGNs with large X-ray column densities (e.g., Shimizu et al. 2018); however, explanations for the existence of such objects have varied widely. One explanation that is consistent with the unified model is that our line of sight grazes the edge of the obscuring torus where the cloud distribution is less dense, but still provides significant X-ray absorption due to the X-ray corona's small physical size compared to the BLR. This would result in a larger effective covering fraction of the compact corona in comparison with the more extended BLR. This scenario is supported from our X-ray modeling with Borus, where we found that a geometry in which the torus is viewed through the rim provided the best fit to the data.

If we model the torus to have a clumpy distribution (Krolik \& Begelman 1988), then another possibility is that a clump has entered our line of sight during the X-ray observation, causing a temporary increase in the X-ray column density. However, the lack of variability in $N_{\mathrm{H}}$ between epochs of X-ray observations appears to disfavor such short-timescale variability as the cause of the X-ray/optical mismatch (see also Section 4.1).

High levels of X-ray obscuration but lack of optical obscuration could also be explained by the presence of highdensity, ionized gas outflows. For example, the broad-line radio galaxy 3C 445 is classified as Type 1 from broad $\mathrm{H} \alpha$ and $\mathrm{H} \beta$ lines in its optical spectrum; however, Suzaku and Chandra observations show it to be heavily absorbed with $N_{\mathrm{H}} \sim 10^{23} \mathrm{~cm}^{-2}$. The soft X-ray spectrum of this source is dominated by ionized emission lines (Braito et al. 2011). A mechanism proposed by Braito et al. is that the photoionized, outflowing absorber is associated with a disk wind that is clumpy in nature and located close to the central X-ray source. The clumpy distribution of the absorber enables visible sightlines to the BLR gas. High-resolution X-ray grating 


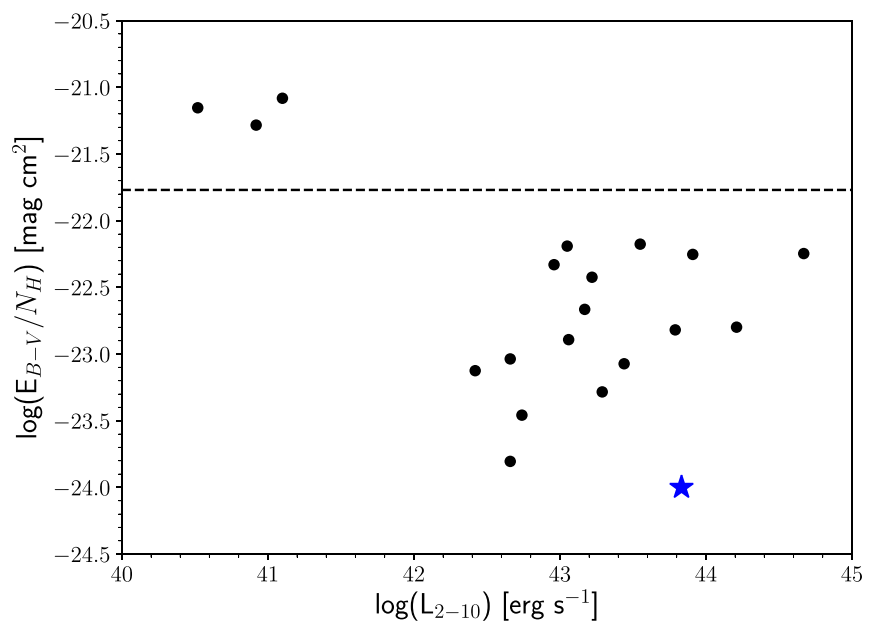

Figure 9. $E_{B-V} / N_{\mathrm{H}}$ ratio vs. intrinsic 2-10 keV X-ray luminosity for 2MASX $\mathrm{J} 193013.80+341049.5$ (blue star), and for sources from the sample of Maiolino et al. (2001) (black points). The reddening $E_{B-V}$ is estimated assuming a Galactic standard extinction curve. The column density $N_{\mathrm{H}}$ is determined from fitting X-ray spectra. The dashed black line represents the Galactic standard value of $E_{B-V} / N_{\mathrm{H}}$.

observations are generally needed to identify emission lines or absorption features associated with ionized gas outflows.

Another possibility for the mismatch in X-ray and optical classifications for 2MASX J193013.80+341049.5 is a lower dust-to-gas ratio relative to the Galactic interstellar medium (ISM). Maiolino et al. (2001) investigated the ratio of reddening to the X-ray-absorbing column density, $E_{B-V} / N_{\mathrm{H}}$, for a diverse sample of AGN characterized by cold X-ray absorption. They found that $E_{B-V} / N_{\mathrm{H}}$ is lower than Galactic by factors ranging from $\sim 3-100$ for most sources in their sample, assuming a standard Galactic extinction curve. In Figure 9, we show $E_{B-V} / N_{\mathrm{H}}$ as a function of the intrinsic $2-10 \mathrm{keV}$ luminosity for 2MASX J193013.80+341049.5 compared to the sample from Maiolino et al. (2001). We find $E_{B-V}=$ $0.50 \pm 0.07$, determined from our broadband SED fitting. We observe that 2MASX J193013.80+341049.5 has an $E_{B-V} / N_{\mathrm{H}}$ that is significantly lower than the Galactic standard value by a factor of 170. This suggests that reduced dust absorption compared to the gaseous column density may likely explain the mismatch between the X-ray and optical classification of 2MASX J193013.80+341049.5. It is possible that the BLR itself provides extra X-ray obscuration and consists of neutral, dust-free gas that is an inner extension of the dusty molecular torus (Davies et al. 2015). If the BLR is the source of the X-ray absorption, $N_{\mathrm{H}}$ variability may be seen on relatively short timescales (e.g., Risaliti et al. 2005). One method to verify where the bulk of the absorbing gas resides is through resolving the width of the $\mathrm{Fe} \mathrm{K} \alpha$ line (e.g., Gandhi et al. 2015). Future X-ray missions such as Athena (Barcons et al. 2015) will be able to measure the width of the $\mathrm{Fe} \mathrm{K} \alpha$ line with unprecedented spectral resolution.

\section{Summary}

In this paper, we present both a broadband X-ray and a multiwavelength analysis of the enigmatic X-ray-obscured, but optically unobscured Type 1 AGN 2MASX J193013.80+341049.5. From joint modeling of NUSTAR and archival XMM-Newton observations, we find 2MASX J193013.80+341049.5 to be strongly absorbed, with $N_{\mathrm{H}}>2 \times 10^{23} \mathrm{~cm}^{-2}$. We also find the source to possess an atypically low coronal temperature compared to the Seyfert population $\left(E_{\text {cut }} \sim 72 \mathrm{keV}\right)$. Some possible mechanisms to explain the low coronal temperature include a large optical depth of the corona, a strong radiation field or a hybrid pair-dominated plasma.

We investigate possible scenarios to explain the mismatch between X-ray and optical classifications using techniques such as broadband SED modeling. We find 2MASX J193013.80 +341049.5 likely has a much lower dust-to-gas ratio relative to the Galactic ISM, with $E_{B-V} / N_{\mathrm{H}}$ lower than the Galactic standard by a factor of $\sim 170$. This suggests that the X-ray/ optical mismatch could be explained by the BLR itself providing the source of extra X-ray obscuration, and being composed of low-ionization, dust-free gas.

We have made use of data from the NUSTAR mission, a project led by the California Institute of Technology, managed by the Jet Propulsion Laboratory, and funded by the National Aeronautics and Space Administration. We thank the NuSTAR Operations, Software and Calibration teams for support with the execution and analysis of these observations. This research has made use of the NUSTAR Data Analysis Software (NuSTARDAS) jointly developed by the ASI Science Data Center (ASDC, Italy) and the California Institute of Technology (USA). M.B. acknowledges support from the Black Hole Initiative at Harvard University, through a grant from the John Templeton Foundation. M.K. acknowledges support from NASA through ADAP award NNH16CT03C. D.J.W. acknowledges support from STFC in the form of an Ernest Rutherford fellowship. R.J.A was supported by FONDECYT grant No. 1191124.

Facilities: NuSTAR, Swift, XMM-Newton, Palomar DBSP.

Software: NuSTARDAS (v2.14.1), HEASOFT (v6.24), XMM SAS (v16.1.0), XSPEC (v12.8.2), Astropy, SciPy, NumPy, Matplotlib.

\section{ORCID iDs}

Nikita Kamraj (iD https://orcid.org/0000-0002-3233-2451

Daniel Stern (iD https://orcid.org/0000-0003-2686-9241

Roberto J. Assef (iD https://orcid.org/0000-0002-9508-3667

Michael J. Koss (i) https://orcid.org/0000-0002-7998-9581

Kyuseok Oh (ii) https://orcid.org/0000-0002-5037-951X

Dominic J. Walton (i) https://orcid.org/0000-0001-5819-3552

\section{References}

Antonucci, R. 1993, A\&A, 31, 473

Arnaud, K. 1996, in ASP Conf. Ser. 101, Astronomical Data Analysis Software and Systems, ed. G. H. Jacoby \& J. Barnes (San Francisco, CA: ASP), 17 Assef, R. J., Kochanek, C. S., Brodwin, M., et al. 2010, ApJ, 713, 970 Baloković, M., Brightman, M., Harrison, F. A., et al. 2018, ApJ, 854, 42 Barcons, X., Nandra, K., Barret, D., et al. 2015, JPhCS, 610, 012008 Baumgartner, W. H., Tueller, J., Markwardt, C. B., et al. 2013, ApJS, 207, 19 Braito, V., Reeves, J. N., Sambruna, R. M., \& Gofford, J. 2011, MNRAS, 414, 2739

Bruzual, G., \& Charlot, S. 2003, MNRAS, 344, 1000

Davies, R. I., Burtscher, L., Rosario, D., et al. 2015, ApJ, 806, 127

Evans, I. N., Ford, H. C., Kinney, A. L., et al. 1991, ApJL, 369, L27

Evans, P. A., Beardmore, A. P., Page, K. L., et al. 2009, MNRAS, 397, 1177

Fabian, A. C., Lohfink, A., Belmont, R., Malzac, J., \& Coppi, P. 2017, MNRAS, 467, 2566

Fiore, F., Puccetti, S., Brusa, M., et al. 2009, ApJ, 693, 447

Gandhi, P., Hönig, S. F., \& Kishimoto, M. 2015, ApJ, 812, 113

Gandhi, P., Horst, H., Smette, A., et al. 2009, A\&A, 502, 457

Gehrels, N., Chincarini, G., Giommi, P., et al. 2004, ApJ, 611, 1005

Ghisellini, G., Haardt, F., \& Fabian, A. C. 1993, MNRAS, 263, L9 
Greene, J. E., \& Ho, L. C. 2007, ApJ, 670, 92

Harrison, F. A., Craig, W. W., Christensen, F. E., et al. 2013, ApJ, 770, 103

Hogg, J. D., Winter, L. M., Mushotzky, R. F., Reynolds, C. S., \& Trippe, M. 2012, ApJ, 752, 153

Kalberla, P. M. W., Burton, W. B., Hartmann, D., et al. 2005, A\&A, 440, 775

Kamraj, N., Harrison, F. A., Baloković, M., Lohfink, A., \& Brightman, M. 2018, ApJ, 866, 124

Kara, E., García, J. A., Lohfink, A., et al. 2017, MNRAS, 468, 3489

Koss, M., Trakhtenbrot, B., Ricci, C., et al. 2017, ApJ, 850, 74

Krolik, J. H., \& Begelman, M. C. 1988, ApJ, 329, 702

Landi, R., Masetti, N., Morelli, L., et al. 2007, ApJ, 669, 109

López-Gonzaga, N., Burtscher, L., Tristram, K. R. W., Meisenheimer, K., \& Schartmann, M. 2016, A\&A, 591, A47

Lusso, E., Comastri, A., Vignali, C., et al. 2010, A\&A, 512, A34

Lusso, E., \& Risaliti, G. 2016, ApJ, 819, 154

Lutz, D., Maiolino, R., Spoon, H. W. W., \& Moorwood, A. F. M. 2004, A\&A, 418, 465

Madsen, K. K., Harrison, F., Broadway, D., et al. 2018, Proc. SPIE, 10699, $106996 \mathrm{M}$

Magdziarz, P., \& Zdziarski, A. A. 1995, MNRAS, 273, 837

Maiolino, R., Marconi, A., Salvati, M., et al. 2001, A\&A, 365, 28

Marinucci, A., Bianchi, S., Matt, G., et al. 2016, MNRAS, 456, L94

Merloni, A., Bongiorno, A., Brusa, M., et al. 2014, MNRAS, 437, 3550

Murphy, K. D., \& Yaqoob, T. 2009, MNRAS, 397, 1549

Oh, K., Koss, M., Markwardt, C. B., et al. 2018, ApJS, 235, 4

Oh, K., Sarzi, M., Schawinski, K., \& Yi, S. K. 2011, ApJS, 195, 13
Oh, K., Yi, S. K., Schawinski, K., et al. 2015, ApJS, 219, 1

Panessa, F., \& Bassani, L. 2002, A\&A, 394, 435

Panessa, F., Bassani, L., Cappi, M., et al. 2006, A\&A, 455, 173

Perri, M., Puccetti, S., \& Spagnuolo, N. 2017, The NuSTAR Data Analysis Software Guide, v1.9.3, https://heasarc.gsfc.nasa.gov/docs/nustar/analysis/ nustar_swguide.pdf

Ricci, C., Trakhtenbrot, B., Koss, M. J., et al. 2017, ApJS, 233, 17

Risaliti, G., Elvis, M., Fabbiano, G., Baldi, A., \& Zezas, A. 2005, ApJL, 623, L93

Sánchez, S. F., García-Lorenzo, B., Jahnke, K., et al. 2006, AN, 327, 167

Shimizu, T. T., Davies, R. I., Koss, M., et al. 2018, ApJ, 856, 154

Stern, D. 2015, ApJ, 807, 129

Storchi-Bergmann, T., Wilson, A. S., \& Baldwin, J. A. 1992, ApJ, 396, 45

Strüder, L., Briel, U., Dennerl, K., et al. 2001, A\&A, 365, L18

Tortosa, A., Marinucci, A., Matt, G., et al. 2017, MNRAS, 466, 4193

Tran, H. D., Lyke, J. E., \& Mader, J. A. 2011, ApJL, 726, L21

Trippe, M. L., Reynolds, C. S., Koss, M., Mushotzky, R. F., \& Winter, L. M. 2011, ApJ, 736, 81

Tristram, K. R. W., Meisenheimer, K., Jaffe, W., et al. 2007, A\&A, 474, 837

Turner, M. J. L., Abbey, A., Arnaud, M., et al. 2001, A\&A, 365, L27

Urry, C. M., \& Padovani, P. 1995, PASP, 107, 83

Verner, D. A., Ferland, G. J., Korista, K. T., \& Yakovlev, D. G. 1996, ApJ, 465,487

Walton, D. J., Tomsick, J. A., Madsen, K. K., et al. 2016, ApJ, 826, 87

Wilms, J., Allen, A., \& McCray, R. 2000, ApJ, 542, 914

Winter, L. M., Lewis, K. T., Koss, M., et al. 2010, ApJ, 710, 503

Zdziarski, A. A., Zycki, P. T., \& Krolik, J. H. 1993, ApJL, 414, L81 